\title{
COSTO-EFECTIVIDAD DE DOS TERAPIAS ANTIVIRALES PARA HEPATITIS B CRÓNICA EN EL PERÚ: ENTECAVIR Y TENOFOVIR
}

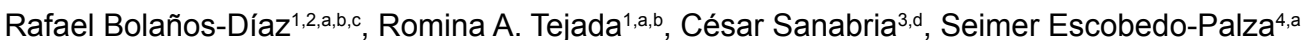

\begin{abstract}
RESUMEN
Objetivos. Comparar en términos de costo-efectividad a entecavir (ETV) y tenofovir (TDF) en el tratamiento del virus de la hepatitis B (HBV) en hospitales públicos del Perú. Materiales y métodos. Estructuramos un modelo de Markov, definimos la efectividad en años de vida ajustados a calidad (AVAC). Incluimos los costos directos del tratamiento en soles desde la perspectiva del Ministerio de Salud del Perú. Calculamos la relación entre costo y efectividad incrementales (ICER). Realizamos análisis de sensibilidad determinístico y probabilístico, considerando un rango de disponibilidad de pago (WTP) desde uno hasta tres veces el producto bruto interno (PBI) per-cápita, y el beneficio monetario neto (BMN) o ICER en el caso del análisis de tornado. Resultados. El tratamiento con TDF es más efectivo y menos costoso que ETV. El ETV tuvo un costo por AVAC de S/ 4482, y de S/ 1526 para TDF. El TDF mantiene un BMN progresivamente mayor conforme aumenta la WTP. La tasa de descuento fue la única variable con efecto significativo en la incertidumbre del modelo. Conclusiones. El tratamiento con TDF es más costo-efectivo que ETV en hospitales públicos del Perú.
\end{abstract}

Palabras clave: Hepatitis B, antiretrovirales, Economía médica. (fuente: DeCS BIREME).

\section{COST-EFFECTIVENESS OF TWO ANTIVIRAL THERAPIES FOR CHRONIC HEPATITIS B IN PERU: ENTECAVIR AND TENOFOVIR}

\begin{abstract}
Objetives. To compare in terms of cost-effectiveness to entecavir (ETV) and tenofovir (TDF) in the treatment of hepatitis B virus (HBV) in public hospitals in Peru. Materials and methods. We structured a Markov model. We define effectiveness adjusted life years for quality (QALY). We include the direct costs of treatment in soles from the perspective of the Ministry of Health of Peru. We estimate the relationship between cost and effectiveness ratios (ICER). We performed sensitivity analyzes considering a range of willingness to pay (WTP) from one to three times the Gross Domestic Product (GDP) per capita, and a tornado analysis regarding Monetary Net Profit (BMN) or ICER. Results. Treatment with TDF is more effective and less expensive than ETV. The ETV had a cost per QALY of PEN 4482, and PEN 1526 TDF. The PTO maintains a progressively larger with increasing WTP BMN. The discount rate was the only variable with a significant effect on model uncertainty. Conclusion: Treatment with TDF is more cost-effective than ETV in public hospitals in Peru.
\end{abstract}

Key words: Hepatitis B; Anti-Retroviral Agents; Economics, Medical. (source: MeSH NLM).

\section{INTRODUCCIÓN}

El virus de la hepatitis B (HBV) pertenece a la familia Hepadnaviridae (ortohepadnavirus), y puede ocasionar tanto enfermedad aguda como crónica. Existen diez genotipos (A-J) que difieren en un $8 \%$, y múltiples subgenotipos que difieren en un $4 \%{ }^{(1,2)}$. La transmisión es porvía sanguínea, mediante relaciones sexuales, por procedimientos médicos con material o productos contaminados y, en menor medida, por vía transplacentaria ${ }^{(3)}$.

El diagnóstico se realiza mediante la detección del antígeno superficial ( $\mathrm{HbsAg})$, en los casos de infección aguda se detectará además inmunoglobulina $\mathrm{M}(\lg \mathrm{M})$ contra el antígeno del núcleo ( $\mathrm{HbcAg})$. Al inicio de la infección los pacientes también son seropositivos para

\footnotetext{
Unidad de Análisis y Generación de Evidencias en Salud Pública. Centro Nacional de Salud Pública. Instituto Nacional de Salud. Lima, Perú. Organización Médica para el desarrollo de la Salud. Lima, Perú.

Unidad de Posgrado de Economía. Universidad Nacional Mayor de San Marcos. Lima, Perú.

Sociedad Peruana de Administración de Salud. Lima, Perú.

Médico cirujano; ${ }^{\mathrm{b}}$ magíster en Epidemiología clínica; ${ }^{\mathrm{c}}$ magíster en Economía de la Salud; ${ }^{\mathrm{d}}$ economista

Trabajo presentado como poster en ISPOR 21st Annual International Meeting en Washington DC.

Recibido: 14/11/2016 Aprobado: 23/08/2017 En línea: 29/09/2017
}

Citar como: Bolaños-Díaz R, Tejada RA, Sanabria C, Escobedo-Palza S. Costo-efectividad de dos terapias antivirales para hepatitis B crónica en el Perú: entecavir y tenofovir. Rev Peru Med Exp Salud Publica. 2017;34(3):377-85. doi: 10.17843/rpmesp.2017.343.2496 
el antígeno e del HBV (HbeAg). La infección crónica se define por la presencia de HbsAg por seis o más meses y la ausencia de $\lg M$ anti-Hbc ${ }^{(4,5)}$.

La sintomatología de la infección aguda por el HBV es muy variada, yendo desde formas asintomáticas hasta formas severas como insuficiencia hepática aguda o fulminantes. Por otro lado, la infección crónica suele llevar a cirrosis y carcinoma hepatocelular; esto último es más frecuente cuando la infección se adquiere en la infancia, es así que el $25-50 \%$ de niños infectados entre el primer y quinto año desarrollarán infección crónica, mientras que cuando la infección ocurre en adolescentes o adultos dicha probabilidad será del $6-10 \%$, de los cuales el $15 \%$ desarrollará cirrosis o carcinoma hepatocelular. Lamentablemente, los pacientes con infección crónica no suelen presentar síntomas hasta bastante avanzada la enfermedad, lo que demora su tratamiento (4).

Según datos de la Organización Mundial de Salud (OMS) existen 248 millones de personas con infección crónica por el HBV ${ }^{(6,7)}$, y mueren 786000 personas como consecuencia del HBV ${ }^{\left({ }^{8}\right)}$. Schweitzer et al. reportan una seroprevalencia mundial de $\mathrm{HbsAg}$ de $3,6 \%$, siendo África y la zona del Pacífico oeste las regiones más afectadas con seroprevalencias de HbsAg de 8,8 y 5,3\%, respectivamente ${ }^{(7)}$. En América Latina se estima que hay 7 a 12 millones de personas infectadas con el HBV, con elevadas prevalencias en población indígena de la cuenca del Amazonas, superiores a $8 \%{ }^{(2,6)}$. Así mismo, al año 2013 , se ha estimado una tasa de mortalidad por el HBV de 0,96 por 100000 personas ${ }^{(9)}$. Además, el HBV es responsable de la mitad de las muertes por carcinoma hepatocelular (5,2 por 100000 personas) y de la tercera parte $(4,8$ por 100000$)$ de las muertes por cirrosis ${ }^{(8)}$. En Latinoamérica se ha estimado una tasa de mortalidad por HBV de 0,26 por 100000 personas ${ }^{(9)}$; $y$ que los genotipos más frecuentes son el $\mathrm{F}$ y el $\mathrm{H}^{(2)}$.

En el Perú existen reportes de seroprevalencia de infección por HBV en diversas poblaciones. Se ha reportado en donantes de sangre (2013) que el 0,38\% tenía positividad para $\mathrm{HbsAg}$ y el $4,2 \%$ para anticuerpos anti-HbcAg ${ }^{(10)}$. Chacaltana et al. reportan en personal militar una seroprevalencia de $\mathrm{HbsAg}$ de 0,33 , y $0,58 \%$ para anticuerpos anti-Hbc (11). Dos estudios en Abancay, que incluyeron estudiantes universitarios y donantes de sangre en un hospital público, reportan frecuencias de HbsAg del 2,5 y 1,9\%, respectivamente, y de anticuerpos anti-HbcAg del 28,3 y $35 \%{ }^{(12,13)}$. Finalmente, se ha reportado que la población indígena de la Amazonía sería una de las más afectadas, con una seroprevalencia de HbsAg de $2,1 \%$ en gestantes y $4 \%$ en sus parejas, así como una seroprevalencia de anticuerpos anti-HbcAg de $42,1 \%$ en gestantes y $54,1 \%$ en sus parejas ${ }^{(14)}$.

\section{MENSAJES CLAVE}

Motivación para realizar el estudio. La infección por el virus de la hepatitis B (HBV) puede ocasionar tanto enfermedad aguda como crónica; esta última suele llevar a cirrosis y carcinoma hepatocelular. Existen dos tipos de tratamiento para la infección crónica por HBV, el interferón y los análogos de nucleósido/nucleótido, entre estos últimos se encuentran tenofovir (TDF) y entecavir (ETV). Sin embargo, debido a sus elevados costos es necesario realizar estudios costo-efectividad antes de decidir sobre su cobertura nacional.

Principales hallazgos. El tratamiento con TDF resulta más eficaz y menos costoso llegando a dominar a la intervención con ETV. El costo por AVAC para ETV fue de S/ 4 482, y para el TDF fue de S/ 1526 . El modelo fue sensible únicamente a la tasa de descuento.

Implicancias. Teniendo en consideración una disponibilidad de pago igual a un producto bruto interno per-cápita, el tratamiento con TDF, es muy costo-efectivo en hospitales públicos del Perú.

La OMS considera a la vacunación como principal estrategia de lucha contra la infección por el HBV ${ }^{(6)}$. La vacuna contra el HBV está disponible desde 1982, y tiene una eficacia superior a $95 \%{ }^{(2)}$. Por ello, ha sido incluida en el esquema nacional de inmunizaciones, en un inicio en zonas endémicas y, posteriormente, en toda la población ${ }^{(15)}$. Sin embargo, dicha inclusión ha sido reciente, por lo que aún existe población adulta no protegida y en riesgo de contraer la infección. El tratamiento de la infección crónica busca demorar el avance a cirrosis, reducir la incidencia de cáncer hepatocelular y mejorar la supervivencia a largo plazo. En dicho sentido, se han propuesto diversos tratamientos de la infección crónica con resultados variados. EI tratamiento consiste en dos tipos de drogas, el interferón y los análogos de nucleósidos/nucleótidos (16). Una revisión sistemática reportó la eliminación de HbsAg y HbeAg, así como la normalización de enzimas hepáticas con medicamentos como interferón- $2 \beta$, lamivudina y adenofovir ${ }^{(17)}$. En revisiones sistemáticas que comparan entecavir (ETV) y tenofovir (TDF) con otros análogos de nucleósidos/nucleótidos se ha observado que los pacientes tratados con dichos medicamentos tenían mayores tasas de niveles indetectables de DNA viral, seroconversión de anti-Hbe, negativización de HbeAg, mejoría de la histología hepática, y normalización de los niveles de transaminasas ${ }^{(16)}$.

Es importante recalcar que el tratamiento es prolongado (48 a 72 semanas); además, muchos de estos tratamientos son costosos, por lo que no han sido aún implementados en varios países como el Perú. En dicho sentido, la OMS recomienda que se dé prioridad a aquellas personas con un estadio más avanzado de enfermedad hepática y con mayor riesgo de morir. Así mismo, recomienda que se empleen análogos de nucleósidos con baja farmacorresistencia como son el TDF y el ETV (18). Ambos medicamentos tienen como ventaja el tomarse de manera oral, una vez al día y pocos 
eventos adversos reportados. Por lo anteriormente expuesto, el presente estudio buscó determinar la relación de costo-efectividad entre ambos tratamientos de la infección crónica por el HBV.

\section{MATERIALES Y MÉTODOS}

\section{DISEÑO DEL ESTUDIO}

Estructuramos un modelo de cadenas de Markov para las siguientes dos estrategias de intervención: 1) ETV y 2) TDF. Para cada estrategia determinamos cuatro estados temporales de enfermedad: "HBV-crónica en tratamiento.", "respuesta viral sostenida" (RVS), "cirrosis compensada" (CC) y "carcinoma hepatocelular" (CHC) y dos estados absorbentes: "terapia de segunda línea" y "muerte". Ubicamos al 100\% de la cohorte hipotética en un estado temporal inicial de "HBV-crónica en tratamiento", con dos desenlaces probables: RVS o falla al tratamiento. Consideramos que toda RVS puede tener los siguientes desenlaces en el transcurso de cada ciclo markoviano: 1) recaída, 2) continuar en RVS, 3) desarrollar CC, 4) desarrollar $\mathrm{CHC}$, o 5) morir por causa hepática (Figura 1).

De acuerdo con la mayor parte de reportes, consideramos la tasa de RVS durante el primer ciclo de Markov (un año) según el estado serológico del HbeAg. Posterior al primer año de tratamiento se asumió que toda recaída transcurre al estado absorbente "terapia de segunda línea". Asimismo, el estado de enfermedad CC transcurre por tres desenlaces probables: "cirrosis descompensada" (CD), CHC o "continuar como
CC". Para el estado $\mathrm{CHC}$ se definió únicamente dos desenlaces probables: "sobrevida" o "muerte".

Según opinión de expertos locales, la edad de la cohorte inicial se estableció en 40 años por considerarse la edad promedio de diagnóstico más frecuente. De igual modo, asumimos un tratamiento de 48 y 72 semanas de duración por considerarse lo más frecuente en nuestro medio.

Para evaluar los resultados calculamos la relación costoefectividad (C-E) de cada intervención, considerándose como más eficiente aquella con el valor más bajo. También calculamos la relación entre el costo incremental y la efectividad incremental (Incremental Cost-Effectiveness Ratio [ICER]), lo cual permitió conocer el costo necesario por año de vida ajustado a calidad (AVAC) para cada una de ellas.

Finalmente, definimos un horizonte temporal de diez años desde la perspectiva del Ministerio de Salud (MINSA), y establecimos la duración del ciclo markoviano en un año. Para el horizonte temporal considerado, aplicamos una tasa de descuento de $3 \%$ tanto a los costos como a las utilidades del modelo. Utilizamos para el análisis el software TreeAge, versión 2015.

\section{EFECTIVIDAD}

Realizamos búsquedas sistemáticas para detectar ensayos clínicos comparativos entre estos dos tratamientos. Se analizaron los datos de eficacia de cada tratamiento reportados en la Guía Clínica de la OMS ${ }^{(3)}$, para contrastar

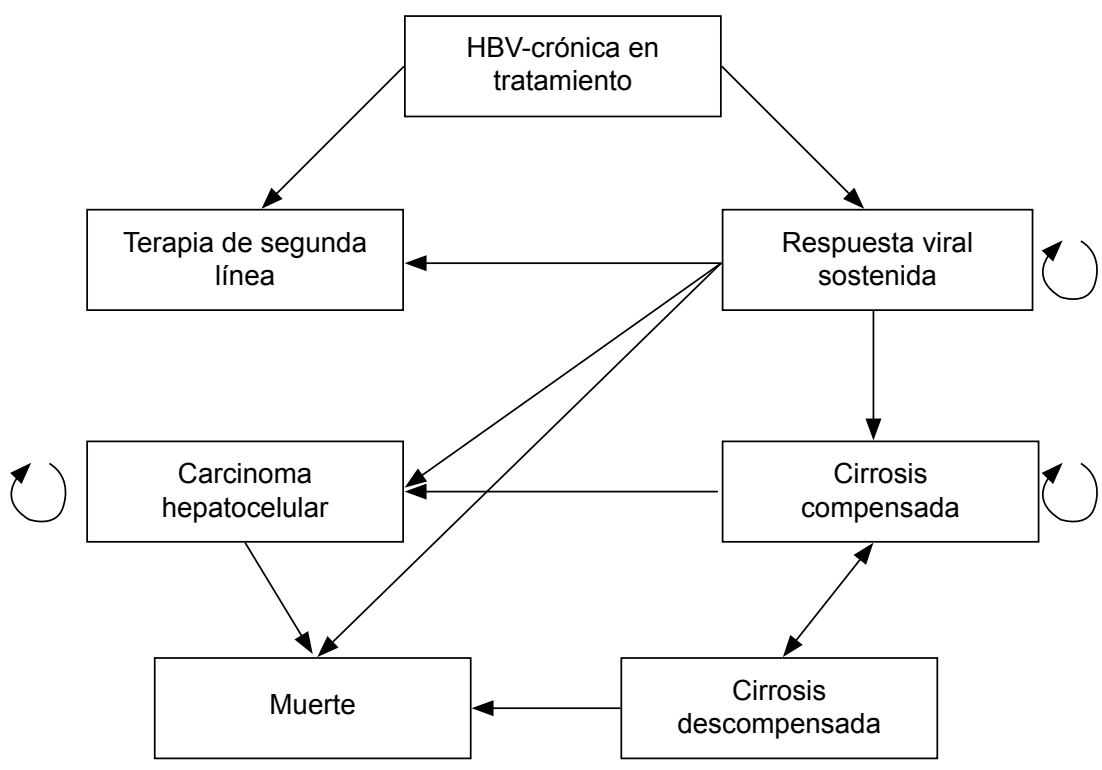

Figura 1. Modelo Markov de costo-efectividad del tratamiento de infección por el virus hepatitis B 
dicha información con la obtenida de expertos locales. La efectividad se definió en AVAC. Todas las probabilidades de transición fueron definidas bajo una distribución beta (rango 0 a 1) teniendo en cuenta sus valores de intervalo asumidos en el modelo para cada una de ellas. Para las utilidades y disutilidades del modelo se asumieron distribuciones normales.

Debido a la ausencia de datos locales se tomaron datos de calidad de vida reportados en estudios C-E previos que habían considerado AVAC para algunos estados de enfermedad comunes a nuestro modelo económico, como RVS, CC, CD y CHC. De igual modo, fue necesario extrapolar algunas probabilidades de transición reportadas en estudios previos para la estimación de algunos desenlaces en nuestro modelo (Tabla 1).

\section{ANÁLISIS DE COSTOS}

Después de definir los flujogramas de atención para los distintos escenarios clínicos contrastando las Guías de Atención de la OMS ${ }^{(3)}$ y la opinión de expertos locales, estimamos los costos con la información brindada por los centros de costos de hospitales del MINSA y de EsSalud (en caso de no contar con datos del MINSA), en base a costos unitarios al año 2015. La perspectiva de los costos es del financiador interesado (MINSA); por lo tanto, para el análisis solo se incluyeron los costos directos del tratamiento. Los costos fueron calculados en soles (S/) al 2015. Se determinó una distribución Log-Normal para todos los costos considerando su mayor tendencia a subir que a bajar. Los costos tuvieron una distribución log-normal.

\section{ANÁLISIS DE SENSIBILIDAD}

Luego de calcular el ICER y el costo por AVAC, se procedió al análisis de tornado para detectar la(s) variable(s) con mayor efecto potencial en el resultado esperado del modelo con respecto al beneficio monetario neto (BMN) o ICER y considerando una disponibilidad de pago (Willingness To Pay [WTP]) de un producto bruto interno (PBI) per-cápita anual (USD 6660).

De acuerdo a las distribuciones probabilísticas incorporadas al modelo, se procedió al análisis de simulación de Montecarlo (1000 simulaciones) para obtener la distribución

Tabla 1. Probabilidades de transición y distribución para los diferentes desenlaces en el tratamiento de la infección por HBV.

\begin{tabular}{|c|c|c|c|c|c|}
\hline Estado inicial & Estado resultante & $\begin{array}{c}\text { Probabilidad de } \\
\text { transición } \mathrm{HBeAg}(+)\end{array}$ & $\begin{array}{c}\text { Probabilidad de } \\
\text { transición } \mathrm{HBeAg}(-)\end{array}$ & Distribución ${ }^{* *}$ & Referencia \\
\hline \multirow[t]{5}{*}{ HBV } & RVS (TDF) & 0,941 & 0.976 & tunnel & (17) \\
\hline & RVS (ETV) & 0,645 & 0.919 & tunnel & (17) \\
\hline & Continúa en RVS (TDF) & 0,790 & 0.790 & $\beta(40 ; 16)$ & (18) \\
\hline & Continúa en RVS (ETV) & 0,720 & 0.720 & $\beta(40 ; 11)$ & (18) \\
\hline & Cirrosis (TDF, ETV) & 0,016 & 0.005 & _tunnel & (19) \\
\hline \multirow[t]{4}{*}{ RVS } & $\mathrm{CHC}(\mathrm{TDF})^{*}$ & 0,$014 ; 0,024$ & 0,$014 ; 0,024$ & _stage & (17) \\
\hline & $\mathrm{CHC}(\mathrm{ETV})^{*}$ & 0,$039 ; 0,066$ & 0,$039 ; 0,066$ & _stage & (17) \\
\hline & Muerte (TDF)* & 0,$007 ; 0,014$ & 0,$007 ; 0,014$ & _stage & (17) \\
\hline & Muerte $(\mathrm{ETV})^{*}$ & 0,$030 ; 0,038$ & 0,$030 ; 0,038$ & _stage & (17) \\
\hline \multirow[t]{2}{*}{$\mathrm{CC}$} & CD (TDF, ETV) & 0,014 & 0,014 & $\beta(1 ; 150)$ & (19) \\
\hline & CHC (TDF, ETV) & 0,023 & 0,023 & $\beta(4 ; 165)$ & (19) \\
\hline CD & Muerte (TDF, ETV) & 0,156 & 0,156 & $\beta(10 ; 50)$ & (19) \\
\hline $\mathrm{CHC}$ & Muerte (TDF, ETV) & 0,560 & 0,560 & $\beta(20 ; 16)$ & (19) \\
\hline Edad (40 años) & & 0,87 & & $\mathrm{~N}(0,87 ; 0,5)$ & (20) \\
\hline Hepatitis viral B (HBV) & & 0,87 & & $\mathrm{~N}(0,87 ; 0,5)$ & (21) \\
\hline $\begin{array}{l}\text { Respuesta viral } \\
\text { sostenida (RVS) }\end{array}$ & & 0,87 & & $N(0,87 ; 0,5)$ & (21) \\
\hline $\begin{array}{l}\text { Cirrosis compensada } \\
\text { (CC) }\end{array}$ & & 0,81 & & $N(0,81 ; 0,5)$ & (21) \\
\hline $\begin{array}{l}\text { Cirrosis } \\
\text { descompensada (CD) }\end{array}$ & & 0,49 & & $N(0,49 ; 0,5)$ & (21) \\
\hline $\begin{array}{l}\text { Carcinoma } \\
\text { hepatocelular }(\mathrm{CHC})\end{array}$ & & 0,85 & & $N(0,85 ; 0,5)$ & (21) \\
\hline
\end{tabular}

HBV: hepatitis viral B; RVS: respuesta viral sostenida; CC: cirrosis hepática; CD: cirrosis descompensada, CHC: carcinoma hepatocelular; TDF: tenofovir; ETV: entecavir.

* Tasa a 3 y 5 años respectivamente. Se asumió el mismo dato para ambas condiciones serológicas ( $\mathrm{HbeAg}+$ y HbeAg-).

**Algunas probabilidades transicionales han sido definidas como funciones en el programa TreeAge, es decir, cambian según la clasificación serológica (_tunnel) o cambian según el ciclo markoviano indicado (_stage). Dichas probabilidades no tienen distribución característica. 
de las nubes de puntos correspondientes al análisis probabilístico. Una vez corrida la simulación, se procedió a graficar las curvas de aceptabilidad para las dos estrategias de tratamiento, considerando un rango de WTP desde S/ 0 hasta el valor de tres PBI per-cápita anual.

Segraficóla ubicación de las nubes de puntos incrementales en el plano bidimensional (gradiente de costos [eje "y"] y gradiente de efectividad [eje " $x$ "] en relación a la recta de disponibilidad de pago. Con dicho gráfico se confrontaron ambos tratamientos. Finalmente, para las dos estrategias de tratamiento se graficaron los cambios en el BMN con respecto a un rango de WTP desde S/ 0 hasta dos PBIs.

El presente reporte sigue las recomendaciones del Consolidated Health Economic Evaluation Reporting Standards (CHEERS), elaborado por la Sociedad Internacional de Facrmacoeconomía e Investigación de Resultados (ISPOR) ${ }^{(19)}$.

\section{CONSIDERACIONES ÉTICAS}

Al ser el presente un estudio económico con poblaciones hipotéticas y datos secundarios, no requiere evaluación de un comité de ética.

\section{RESULTADOS}

La composición del costo para los tratamientos está dada, principalmente, por las consultas especializadas, la batería de pruebas (incluyendo la carga viral) y la medicación antiviral. El costo promedio del tratamiento semanal para el TDF se estimó en S/ 112,78 (USD 33) dando un costo promedio total anual de S/ 5 413,28 (USD 1 587). De igual modo, para la opción ETV, el costo promedio semanal se estimó en S/ 192,44 (USD 56) con un costo promedio total anual de S/ 9236,96 (USD 2709). El costo promedio de las principales complicaciones fue estimado uno por uno, pero se estimó para el conjunto de las mismas un costo total ponderado de S/ 2204 (USD 646); siendo este último valor considerado para el presente modelo (Tabla 2).

\section{RESULTADOS DEL CASO BASE}

El análisis determinístico primario muestra que el tratamiento con TDF resulta más eficaz y menos costoso, llegando a dominar a la intervención con ETV, tanto en el tratamiento de 48 como de 72 semanas (Tabla 3). El costo por AVAC para ETV fue de S/ 4482 (USD 1314) en el tratamiento de 48 semanas, y de S/ 6961 (USD 2041) en el tratamiento de 72 semanas. En el caso de TDF fue de S/ 1526 (USD 448) tratamiento de 48 semanas y de S/ 2128 (USD 624) en el tratamiento de 72 semanas (Tabla 3).

\section{ANÁLISIS DE SENSIBILIDAD}

La simulación de Montecarlo (1000 simulaciones) dibuja dos nubes de puntos (una para cada estrategia)

Tabla 2. Costos del tratamiento de la infección crónica por HBV con tenofovir y entecavir

\begin{tabular}{lccc}
\hline Componente del costo & Costo tratamiento anual - S/ & Porcentaje & Costo promedio semanal- S/ \\
\hline Consulta externa hepatólogo & 216,00 & 3,99 & 4,50 \\
\hline Prueba de hepatitis (carga viral HBV) & 200,00 & 3,69 & 4,17 \\
\hline Tenofovir (300 mg/día) & 1387,68 & 25,63 & 28,91 \\
\hline Entecavir (0,5 mg/día) & 5211,36 & 56,42 & 108,57 \\
\hline Batería de pruebas & 3609,60 & 66,68 & 112,78 \\
\hline TOTAL Tenofovir & 5413,28 & 100,00 & 192,44 \\
\hline TOTAL Entecavir & 9236,96 & 100,00 & \\
\hline Estadios & Costo promedio (S/) & & \\
\hline Cirrosis compensada & 1000 & & \\
\hline Cirrosis descompensada por & & & \\
\hline Sangrado ulceroso & 1033 & & \\
\hline Sangrado variceal & 6537 & & \\
\hline Infección de tejidos blandos & 1440 & & \\
\hline Neumonía & 1685 & & \\
\hline Infección urinaria & 1051 & & \\
\hline Peritonitis & 1481 & & \\
\hline Total ponderado* & 2204 & & \\
\hline
\end{tabular}

* El cálculo del costo de la cirrosis descompensada fue ponderado en base a la frecuencia de las complicaciones. 
en el plano bidimensional, las que se van distanciando hacia la derecha superponiéndose parcialmente en zonas marginales. No obstante, la dispersión de puntos correspondiente a la intervención ETV se ubica como menos efectiva pero más costosa frente a la opción TDF, tanto en el tratamiento de 48 como en el de 72 semanas (Figura 2).

Como es de esperarse, por la dominancia ejercida por TDF, las curvas de aceptabilidad de ambos tratamientos muestran a éste como más costo-efectivo a lo largo de todo el intervalo de WTP (datos no mostrados). La intervención con TDF resulta probabilísticamente más costo-efectiva en comparación con ETV, ubicándose la mayor proporción de puntos por debajo de la recta de WTP (menor a un PBI per-cápita anual), pudiendo ser más efectivo y menos costoso en $79 \%$ de las simulaciones en el tratamiento de 48 semanas y $85 \%$ en el tratamiento de 72 semanas, más efectivo y más costoso en $15 \%$ de las simulaciones en el tratamiento de 48 semanas y $9 \%$ en el tratamiento de 72 semanas, y menos efectivo y menos costoso en $3 \%$ de las simulaciones tratamiento de 48 semanas y $5,4 \%$ en el tratamiento de 72 semanas (Figura 3).

En el análisis del BMN con respecto a la WTP observamos que el TDF mantiene un BMN progresivamente mayor conforme aumenta la WTP, tanto en el tratamiento de 48 semanas como en el de 72 semanas. Finalmente, el análisis multivariado de tornado para ambos periodos de tratamiento detectó a la tasa de descuento (de 0 a
$6 \%$ ) como la única variable crítica con efecto potencial significativo en la incertidumbre de los resultados del modelo, sea con respecto a BMN o ICER.

\section{DISCUSIÓN}

Los resultados del presente análisis sugieren que la $\mathrm{C}-\mathrm{E}$ de la intervención con TDF domina a la opción ETV en el tratamiento de la infección por el HBV. De igual modo, la dispersión de los puntos incrementales de C-E en el plano cartesiano reafirma la mayor C-E del TDF. Otros estudios costo-efectividad del tratamiento de la HBV que comparan TDF y ETV coinciden con nuestro estudio en reportar al TDF como más costo-efectivo ${ }^{(20,21)}$.

Se consideraron 48 semanas de tratamiento para los dos esquemas comparados, teniendo en cuenta que la mayor proporción de pacientes responde en este lapso. No obstante, los resultados mantienen su tendencia cuando se considera un tiempo de tratamiento de 72 semanas.

Nuestra experiencia local coincide con la internacional en la edad promedio de diagnóstico de la HBV crónica (40 años), por lo que se asumió un modelo económico con una cohorte hipotética de esta edad, sin tratamiento previo (pacientes näive) y bajo un horizonte temporal de diez años, considerando que la efectividad en el largo plazo debe consolidarse a futuro con mayor evidencia. Hemos considerado los AVAC según el estado de enfermedad asociado a la edad de diagnóstico ${ }^{(22)}$. De igual modo,
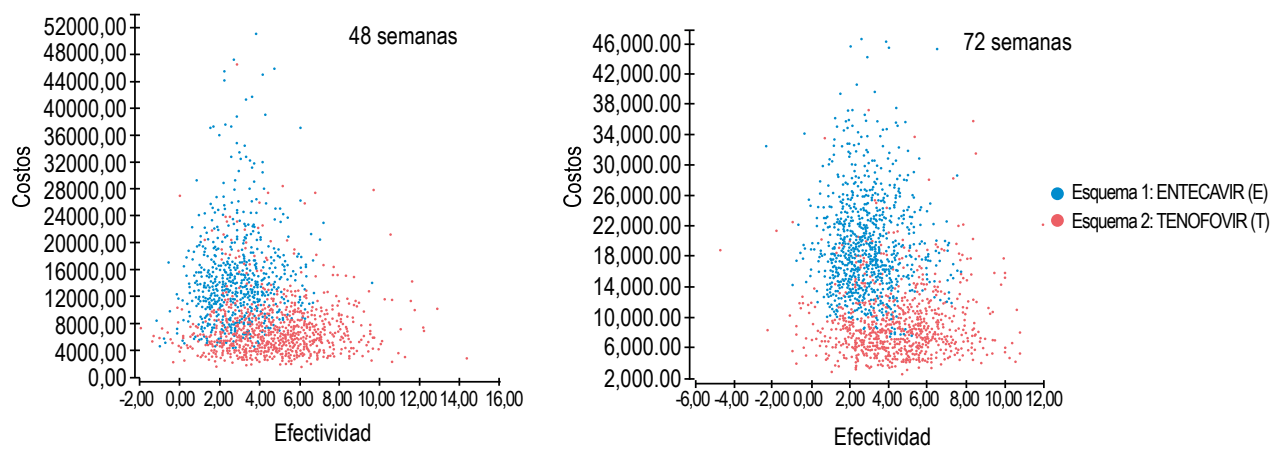

Figura 2. Análisis costo-efectividad probabilístico de dos estrategias de tratamiento de la infección por el HBV.
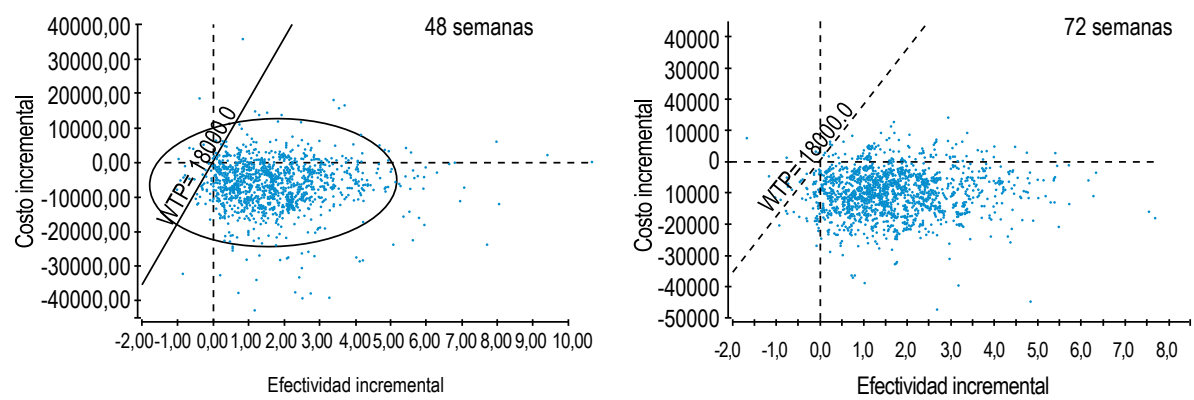

Figura 3. Análisis coto-efectividad incremental del tratamiento de la infección crónica por HBV con tenofovir frente a entecavir. 
Tabla 3. Análisis costo-efectividad determinístico del tratamiento de la infección crónica por el HBV.

\begin{tabular}{|c|c|c|c|c|c|c|c|}
\hline Estrategia & Costo & $\begin{array}{c}\text { Costo } \\
\text { incremental }\end{array}$ & Efectividad & $\begin{array}{c}\text { Efectividad } \\
\text { incremental }\end{array}$ & ICER & BMN & C/E \\
\hline \multicolumn{8}{|l|}{ Tratamiento de 48 semanas } \\
\hline Esquema 2: Tenofovir & 7135 & & 4,68 & & & 77031 & 1526 \\
\hline Esquema 1: Entecavir & 13275 & 6140 & 2,96 & $-1,71$ & -3583 & 40040 & 4482 \\
\hline \multicolumn{8}{|l|}{ Tratamiento de 72 semanas } \\
\hline Esquema 2: Tenofovir & 9491 & & 4,46 & & & 70789 & 2128 \\
\hline Esquema 1: Entecavir & 19267 & 9777 & 2,77 & $-1,69$ & -5780 & 30593 & 6961 \\
\hline
\end{tabular}

ICER: Incremental Cost-Effectiveness Ratio (razón costo-efectividad incremental); BMN: beneficio monetario neto).

el modelo opera bajo un supuesto de que un $40 \%$ de los pacientes cursan con $\mathrm{HbeAg}$ positivo y el resto lo hace con serología negativa a dicho antígeno ${ }^{(23)}$. Esto impacta en el modelo, considerando que, de acuerdo a la guía clínica revisada, los pacientes con $\mathrm{HbeAg}$ negativo (60\%) requieren una mayor dosis de ETV (1 mg/día), en contraste con aquellos pacientes $\mathrm{HbeAg}$ positivos

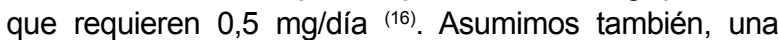
severidad de enfermedad de nivel moderado (F2-F3 de la escala METAVIR) no solo por ser el estado de enfermedad más frecuente al momento del diagnóstico, sino también para equilibrar los resultados de eficacia y seguridad en los grados extremos de enfermedad hepática.

Si bien, se han publicado estudios que comparan directamente ambas opciones de tratamiento ${ }^{(24-31)}$ existen algunas comparaciones indirectas reportadas en distintos metaanálisis en red, que definen una jerarquía en la eficacia de los agentes antivirales ${ }^{(32-34)}$. Para el presente análisis se han considerado los resultados del metaanálisis en red más reciente ${ }^{(18)}$ respecto de las opciones antivirales para HBV crónica, el que comprendió un total 21 pares de comparación con 5073 pacientes näive de tipo HbeAg positivo y 16 pares de comparación con 2604 pacientes näive de tipo $\mathrm{HbeAg}$ negativo. Basados en esta evidencia, la monoterapia con TDF tuvo la mayor probabilidad de alcanzar niveles indetectables de HBV-DNA al final del primer año de tratamiento (48 semanas aproximadamente). Este resultado se observa tanto en aquellos $\mathrm{HbeAg}$ positivo (94,1\%, IC95: 74,7-98,9\%) como en aquellos con $\mathrm{HbeAg}$ negativo (97,6\%, IC95: 56,7-99,9\%). Para la intervención con ETV, los resultados fueron de 64,5\% (IC95: 49,1$80,5 \%$ ) en HbeAg positivo y 91,9\% (IC95: 87,3-95,1\%) en HbeAg negativo, respectivamente.

Por otro lado, a partir de distintas revisiones sistemáticas ${ }^{(35,36)}$, ETV ha demostrado buena respuesta antiviral en pacientes näive con niveles más avanzados de enfermedad (METAVIR F3-F4), aunque la mortalidad no se afecta significativamente. Aún no se han reportado resultados de efectividad con TDF en enfermedad hepática avanzada. Por lo tanto, hasta contar con mayor evidencia a futuro, es recomendable que los decisores consideren que los niveles de enfermedad más leves (METAVIR F1-F2) pueden alcanzar mayores tasas de RVS con menor necesidad de monitoreo clínico, y que los niveles más severos de enfermedad (METAVIR F3-F4) pueden tener menores tasas de RVS asociadas a una mayor necesidad de monitoreo clínico del tratamiento.

Si bien nuestro modelo económico tiene la fortaleza de operar con costos locales, su principal debilidad radica en la transferibilidad de las utilidades a partir de estudios C-E previos ${ }^{(37)}$. Ante la ausencia de datos locales, se buscan datos regionales y cuando estos tampoco están disponibles, se recurre a datos internacionales, como en nuestro caso. Con la finalidad de tomar en cuenta la posibilidad de distintos valores, incluimos rangos amplios en el análisis de sensibilidad para las utilidades, observando que dicha variable no afectaba de manera significativa al modelo. Es por ello que consideramos que no se afectaría la validez del modelo. Igualmente, las probabilidades de transición fueron definidas en base a la revisión bibliográfica, proviniendo en algunos casos de metaanálisis en red, lo cual representaría poblaciones más diversas $\mathrm{y}$, por ende, datos más cercanos a la realidad local. Asimismo, dichas probabilidades fueron consensuadas con la opinión de expertos locales, por lo que consideramos que no afectan la validez del modelo.

Consideramos que sería de utilidad desarrollar estudios de impacto presupuestario (y/o costo-beneficio) para estimar el costo de implementar el tratamiento más costo-efectivo. Igualmente, basándose en los resultados de C-E e impacto presupuestario, será recomendable la ejecución de nuevos análisis con la modificación potencial de los costos (según la disposición del proveedor[es]) con el objetivo de ajustar el ICER al nivel más conveniente posible. Esto en base a o reportado en otros estudios donde disminuciones en el precio del medicamento llevan a modificar la relación de C-E ${ }^{(38)}$.

En conclusión, en el Perú, la intervención con TDF es más costo-efectiva que la opción ETV con un costo aproximado por AVAC de S/ 1526. Probabilísticamente, el tratamiento con ETV queda dominado por el tratamiento con TDF. 
Contribuciones de los autores: RBD y RAT han participado en la concepción y diseño del artículo, recolección de resultados, análisis e interpretación de datos y redacción del artículo. CS y SEP han participado en la recolección de resultados. Todos los autores han realizado la revisión crítica del artículo y aprobación de la versión final.

Fuentes de financiamiento: el estudio fue financiado por el Instituto Nacional de Salud.
Conflictos de interés: Rafael Bolaños-Díaz pertenece también a Organización Médica para el Desarrollo de la Salud (ODDS), consultora privada de investigación biomédica que ha brindado servicios a varias compañías farmacéuticas en Perú; César Sanabria declara haber realizado consultorías para Bristol-Myers Squibb. Romina A. Tejada y Seimer Escobedo-Palza declaran no tener conflictos de interés.

\section{REFERENCIAS BIBLIOGRÁFICAS}

1. Schaefer S. Hepatitis B virus taxonomy and hepatitis B virus genotypes. World $\mathrm{J}$ Gastroenterol. 2007;13:14-21.

2. Alvarado-Mora MV, Pinho JRR. Epidemiological update of hepatitis B, C and delta in Latin America. Antivir Ther. 2013;18:429-33.

3. Organización Mundial de Salud. Guidelines for the prevention, care and treatment of persons with chronic hepatitis B infection [Internet]. Geneva: World Health Organization; 2015 [cited 2015 Nov 19]. Available from: http://www.ncbi.nlm.nih.gov/books/ NBK305553/

4. Mahoney FJ. Update on diagnosis, management, and prevention of hepatitis $B$ virus infection. Clin Microbiol Rev. 1999;12:351-66.

5. Villar LM, Cruz HM, Barbosa JR, Bezerra CS, Portilho MM, Scalioni L de P. Update on hepatitis $\mathrm{B}$ and $\mathrm{C}$ virus diagnosis. World J Virol. 2015;4:323-42.

6. Organización Mundial de la Salud. OMS | Hepatitis B [Internet]. WHO. [cited 2015 Nov 18]. Available from: http:// www.who.int/mediacentre/factsheets/ fs204/es/

7. Schweitzer A, Horn J, Mikolajczyk RT, Krause G, Ott JJ. Estimations of worldwide prevalence of chronic hepatitis $B$ virus infection: a systematic review of data published between 1965 and 2013 . Lancet Lond Engl. 2015;386:1546-55.

8. Lozano R, Naghavi M, Foreman K, Lim S, Shibuya K, Aboyans V, et al. Global and regional mortality from 235 causes of death for 20 age groups in 1990 and 2010: a systematic analysis for the Global Burden of Disease Study 2010. Lancet Lond Engl. 2012;380:2095-128.

9. Institute for Health Metrics and Evaluation. GBD Compare | IHME Viz Hub [Internet]. [cited $2015 \mathrm{Nov}$ 19]. Available from: http://vizhub. healthdata.org/gbd-compare

10. PAHO/WHO. Suministro de sangre para transfusionesen los países de Latinoamérica y del Caribe [Internet]. 2015 [cited 2015
Nov 10]. Available from: http://www. paho.org/hq/index.php?option $=$ com content\&view $=$ article $\& i d=8918 \%$ 3A2013-supply-blood-transfusioncaribbean-latin-american-countries2010-2011\&catid=1163\%3Ahss-bloodservices-\&Itemid=2163\&lang=es

11. Chacaltana A,EspinozaJ. [Seroprevalence of the infection and risk factors of hepatitis $\mathrm{B}$ and $\mathrm{C}$ in healthy military personnel]. Rev Gastroenterol Perú Órgano Of Soc Gastroenterol Perú. 2008;28:217-25.

12. Ramírez-Soto MC, Huichi-Atamari M, Aguilar-Ancori EG, Pezo-Ochoa JD. [Seroprevalence of viral hepatitis $B$ in university students in Abancay, Peru]. Rev Peru Med Exp Salud Pública. 2011;28:513-7.

13. Ramírez-Soto MC, Huichi-Atamari M. [Hepatitis B in blood donors at an hospital in Apurimac, Peru]. Rev Peru Med Exp Salud Pública. 2012;29:163-4.

14. Ormaeche M, Whittembury A, Pun M, Suárez-Ognio L. Hepatitis B virus, syphilis, and HIV seroprevalence in pregnant women and their male partners from six indigenous populations of the Peruvian Amazon Basin, 2007-2008. Int $\mathrm{J}$ Infect Dis IJID Off Publ Int Soc Infect Dis. 2012;16:e724-30.

15. Ministerio de Salud. Norma técnica de salud que establece el esquema nacional de vacunación. RM N510-2013/MINSA [Internet]. 2013 [cited 2013 Dec 17]. Available from: ftp://ftp2.minsa.gob.pe/ normaslegales/2013/RM510_2013_ MINSA.pdf

16. Organización Mundial de la Salud. WHO | Guidelines for the prevention, care and treatment of persons with chronic hepatitis B infection [Internet]. WHO. [cited 2015 Nov 17]. Available from: http://www.who.int/hepatitis/ publications/hepatitis-b-guidelines/en/

17. Shamliyan TA, MacDonald R, Shaukat A, Taylor BC, Yuan J-M, Johnson JR, et al. Antiviral therapy for adults with chronic hepatitis B: a systematic review for a National Institutes of Health Consensus Development Conference. Ann Intern Med. 2009;150:111-24.
18. $\mathrm{WHO} \mid$ Guidelines for the screening, care and treatment of persons with hepatitis C infection [Internet]. WHO. [cited 2015 Nov 9]. Available from: http:// www.who.int/hepatitis/publications/ hepatitis-c-guidelines/en/

19. Husereau D, Drummond M, Petrou S, Carswell C, Moher D, Greenberg D, et al. Consolidated Health Economic Evaluation Reporting Standards (CHEERS)--explanation and elaboration: a report of the ISPOR Health Economic Evaluation Publication Guidelines Good Reporting Practices Task Force. Value Health. 2013;16:231-50.

20. Buti M, Oyagüez I, Lozano V, Casado MA. Cost effectiveness of first-line oral antiviral therapies for chronic hepatitis B : a systematic review. Pharmacoeconomics. 2013;31:63-75.

21. Wiens A, Lenzi L, Venson R, Correr CJ, Rotta I, Pedroso ML, et al. Comparative efficacy of oral nucleoside or nucleotide analog monotherapy used in chronic hepatitis B: a mixedtreatment comparison meta-analysis. Pharmacotherapy. 2013;33:144-51.

22. Sullivan PW, Ghushchyan V. PreferenceBased EQ-5D index scores for chronic conditions in the United States. Med Decis Mak Int J Soc Med Decis Mak. 2006;26:410-20.

23. McMahon BJ. The natural history of chronic hepatitis B virus infection. Semin Liver Dis. 2004;24 Suppl 1:17-21.

24. Doğan ÜB, Kara B, Gümürdülü Y, Soylu A, Akin MS. Comparison of the efficacy of tenofovir and entecavir for the treatment of nucleos $(t)$ idenaive patients with chronic hepatitis B. Turk J Gastroenterol Off J Turk Soc Gastroenterol. 2012;23:247-52.

25. Güzelbulut F, Ovünç AOK, Oetinkaya ZA, Senates E, Gökden Y, Saltürk AGD, et al. Comparison of the efficacy of entecavir and tenofovir in chronic hepatitis B. Hepatogastroenterology. 2012;59:477-80. 
26. Kurdas O, Guzelbulut F. Comparison of the efficacy of Entecavir and Tenofovir in chronic hepatitis B patients with high viral load and/or high fibrosis scores at week 48 of therapy. Gastroenterology. 2011;140(5):S930-1.

27. Gao L, Trinh HN, Li J, Nguyen MH. Tenofovir is superior to entecavir for achieving complete viral suppression in HBeAg-positive chronic hepatitis B patients with high HBV DNA. Aliment Pharmacol Ther. 2014;39:629-37.

28. Liaw Y-F, Sheen I-S, Lee C-M,Akarca US, Papatheodoridis GV, Suet-Hing Wong $\mathrm{F}$, et al. Tenofovir disoproxil fumarate (TDF), emtricitabine/TDF, and entecavir in patients with decompensated chronic hepatitis B liver disease. Hepatol Baltim Md. 2011;53:62-72.

29. Ceylan B, Yardimci C, Fincanci M, Eren G, Tozalgan U, Muderrisoglu C, et al. Comparison of tenofovir and entecavir in patients with chronic HBV infection. Eur Rev Med Pharmacol Sci. 2013;17:2467-73.

30. Sriprayoon T, Lueangarum S, Suwanwela C. Efficacy and safety of Entecavir versus Tenofovir treatment in chronic hepatitis B patients: A randomized controlled trial. Gastroenterology. 2012;1421:S695.

31. Köklü S, Tuna Y, Gülşen MT, Demir M, Köksal AŞ, Koçkar MC, et al. Longterm efficacy and safety of lamivudine, entecavir, and tenofovir for treatment of hepatitis B virus-related cirrhosis. Clin Gastroenterol Hepatol. 2013;11:88-94.

32. Maratea D, Fadda V, Trippoli S, Messori A. Letter: First-line monotherapies for chronic hepatitis B - indirect comparison between entecavir and tenofovir. Aliment Pharmacol Ther. 2013;37:584-5.

33. Mealing S, Ghement I, Hawkins N, Scott DA, Lescrauwaet B, Watt M, et al. The importance of baseline viral load when assessing relative efficacy in treatmentnaïve $\mathrm{HBeAg}$-positive chronic hepatitis $\mathrm{B}$ : a systematic review and network metaanalysis. Syst Rev. 2014;3:21.

34. Govan L, Wu O, Xin Y, Hutchinson SJ, Hawkins N. Comparative effectiveness of antiviral treatment for hepatitis B: a systematic review and Bayesian network meta-analysis. Eur J Gastroenterol Hepatol. 2015;27:882-94.

35. Peng $\mathrm{H}$, Liu J, Yang M, Tong S, Yin W, Tang $\mathrm{H}$, et al. Efficacy of lamivudine combined with adefovir dipivoxil versus entecavir monotherapy in patients with hepatitis B-associated decompensated cirrhosis: A meta-analysis. J Clin Pharmacol. 2014;54:189-200.

36. Ye X-G, Su Q-M. Effects of entecavir and lamivudine for hepatitis $\mathrm{B}$ decompensated cirrhosis: meta-analysis. World J Gastroenterol. 2013;19:6665-78.

37. Bermingham SL, Hughes R, Fenu E, Sawyer LM, Boxall E, T Kennedy P, et al. Cost-Effectiveness Analysis of Alternative Antiviral Strategies for the Treatment of HBeAg-Positive and HBeAg-Negative Chronic Hepatitis B in the United Kingdom. Value Health J Int Soc Pharmacoeconomics Outcomes Res. 2015;18:800-9.

38. Oliveira GLA de, Almeida AM, Silva AL da, Brandão CMR, Andrade EIG, Cherchiglia $\mathrm{ML}$, et al. Incorporated antivirals for chronic hepatitis B in Brazil: a cost-effectiveness analysis. Rev Saúde Pública. 2013;47:769-78; discussion 779.

Correspondencia: Rafael Bolaños Diaz Dirección: Av. Defensores del Morro 2268(Ex Huaylas) Chorrillos, Lima 9

Teléfono: 748-0000 anexo 6650

Correo electrónico: rbolanosd@yahoo.es

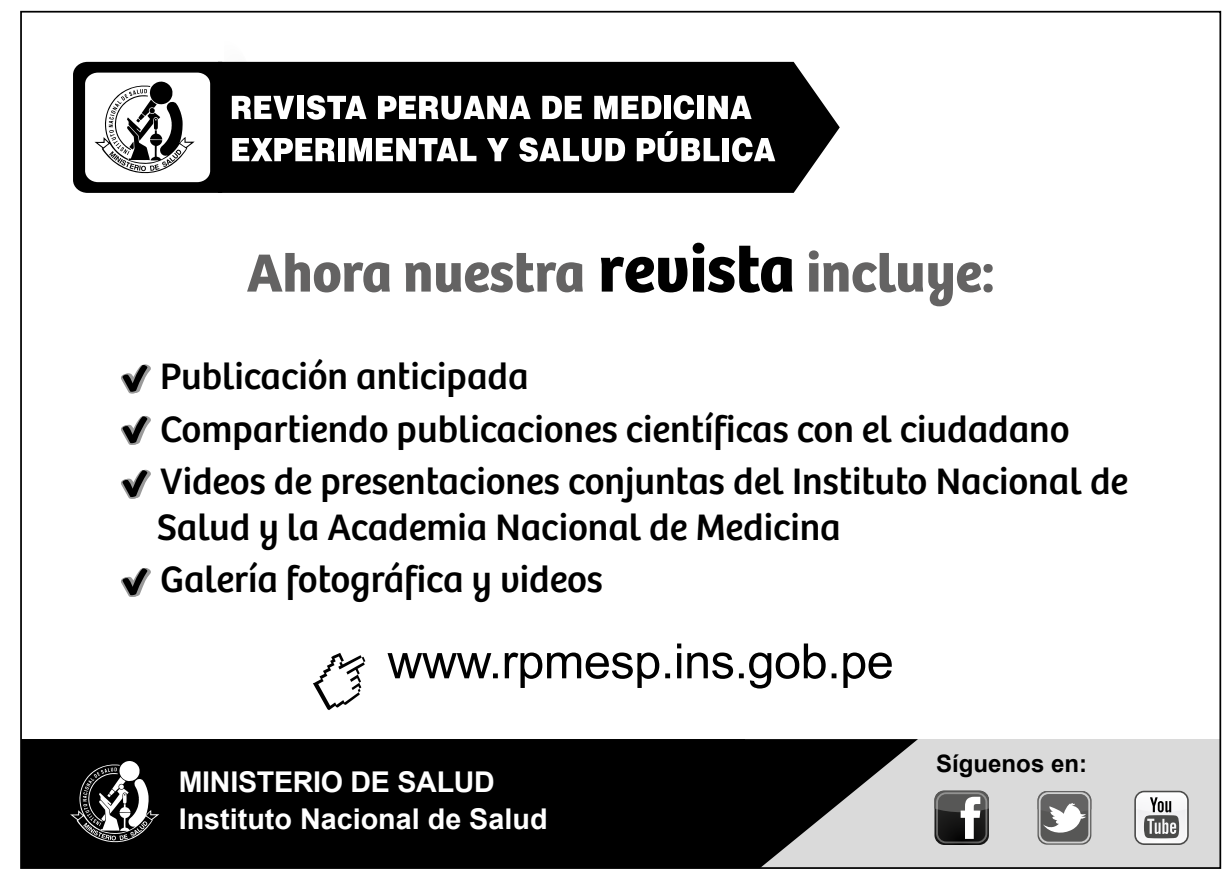

